\title{
Correction to: Imaging of Bronchial Pathology in Antibody Deficiency: Data from the European Chest CT Group
}

Katharina Schütz ${ }^{1}$. Diana Alecsandru年, Bodo Grimbacher ${ }^{3,4}$ - Jamanda Haddock ${ }^{5}$. Annemarie Bruining ${ }^{6}$. Gertjan Driessen $^{7,8}$ • Esther de Vries ${ }^{9,10}$ • Peter M. van Hagen ${ }^{11}$ • leneke Hartmann ${ }^{12}$. Francesco Fraioli ${ }^{13,14}$. Cinzia Milito $^{15} \cdot$ Milica Mitrevski $^{15} \cdot$ Isabella Quinti $^{15} \cdot$ Goffredo Serra $^{13} \cdot$ Peter Kelleher $^{16} \cdot$ Michael Loebinger $^{17}$. Jiri Litzman ${ }^{18}$ • Vera Postranecka ${ }^{19}$ • Vojtech Thon ${ }^{18,20}$. Judith Babar ${ }^{21}$ • Alison M. Condliffe ${ }^{21}$. Andrew Exley ${ }^{22}$. Dinakantha Kumararatne ${ }^{23}$. Nick Screaton ${ }^{24}$. Alison Jones ${ }^{25}$. Maria P Bondioni ${ }^{26}$ - Vassilios Lougaris ${ }^{27}$. Alessandro Plebani ${ }^{27}$. Annarosa Soresina ${ }^{28}$. Cesare Sirignano ${ }^{29}$. Giuseppe Spadaro ${ }^{30}$ • Nermeen Galal $^{31}$. Luis I. Gonzalez-Granado ${ }^{2}$ - Sabine Dettmer ${ }^{32} \cdot$ Robert Stirling $^{33} \cdot$ Helen Chapel $^{34}$ - Mary Lucas ${ }^{34} \cdot$ Smita Patel $^{34}$. Claire-Michele Farber ${ }^{35}$ - Isabelle Meyts ${ }^{36}$. Arpan K Banerjee ${ }^{37}$. Scott Hackett ${ }^{38}$ - John R. Hurst ${ }^{39} \cdot$ Klaus Warnatz $^{4}$. Benjamin Gathmann ${ }^{40} \cdot$ Jürgen Weidemann ${ }^{41}$. Daniel Berthold ${ }^{42} \cdot$ Ulrich Baumann ${ }^{1} \cdot$ for the Chest CT in Antibody Deficiency Group

Published online: 23 March 2019

(C) Springer Science+Business Media, LLC, part of Springer Nature 2019

Correction to: Journal of Clinical Immunology https://doi.org/10.1007/s10875-018-0577-9
Publisher's Note Springer Nature remains neutral with regard to jurisdictional claims in published maps and institutional affiliations.

In the original version of this article unfortunately two authors were missing: Dr. Jürgen Weidemann and Dr. Daniel Berthold. The correct list of authors is presented above.

The online version of the original article can be found at https://doi.org/ 10.1007/s10875-018-0577-9

Ulrich Baumann

Baumann.ulrich@mh-hannover.de

Extended author information available on the last page of the article 


\section{Affiliations}

Katharina Schütz ${ }^{1}$ - Diana Alecsandru ${ }^{2,3}$ • Bodo Grimbacher ${ }^{3,4}$ - Jamanda Haddock ${ }^{5}$ Annemarie Bruining ${ }^{6}$. Gertjan Driessen $^{7,8}$. Esther de Vries ${ }^{9,10}$ • Peter M. van Hagen ${ }^{11}$ • leneke Hartmann ${ }^{12}$ • Francesco Fraioli $^{13,14}$. Cinzia Milito ${ }^{15}$. Milica Mitrevski ${ }^{15} \cdot$ Isabella Quinti $^{15} \cdot$ Goffredo Serra $^{13}$ • Peter Kelleher ${ }^{16}$ Michael Loebinger $^{17}$. $^{16}$ Jiri Litzman ${ }^{18}$. Vera Postranecka ${ }^{19}$ • Vojtech Thon ${ }^{18,20}$ • Judith Babar ${ }^{21}$ • Alison M. Condliffe ${ }^{21} \cdot$ Andrew Exley $^{22}$. Dinakantha Kumararatne ${ }^{23}$. Nick Screaton ${ }^{24}$. Alison Jones ${ }^{25}$. Maria P Bondioni ${ }^{26}$ - Vassilios Lougaris ${ }^{27}$.

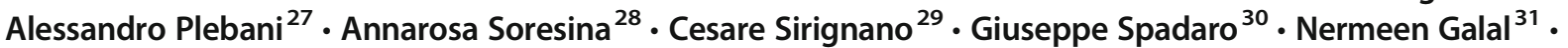
Luis I. Gonzalez-Granado ${ }^{2}$ - Sabine Dettmer ${ }^{32} \cdot$ Robert Stirling $^{33}$ - Helen Chapel ${ }^{34}$. Mary Lucas $^{34} \cdot$ Smita Patel $^{34}$. Claire-Michele Farber ${ }^{35}$ - Isabelle Meyts ${ }^{36}$ - Arpan K Banerjee ${ }^{37}$. Scott Hackett ${ }^{38}$. John R. Hurst ${ }^{39}$ - Klaus Warnatz ${ }^{4}$. Benjamin Gathmann ${ }^{40}$. Jürgen Weidemann ${ }^{41}$. Daniel Berthold ${ }^{42}$ • Ulrich Baumann ${ }^{1}$

1 Paediatric Immunology Unit, Department of Paediatric Pulmonology, Allergology and Neonatology, Hanover Medical School, Carl-Neuberg Str. 1, 30625 Hannover, Germany

2 Primary Immunodeficiencies Unit, Pediatrics, Hospital 12 Octubre, Madrid, Spain

3 Clinical Immunology, Royal Free Hospital, London, UK

4 Centre for Chronic Immunodeficiency, University Medical Center of Freiburg, Freiburg, Germany

5 Radiology, Royal Free Hospital, London, UK

6 Dutch Cancer Institute, Antoni van Leeuwenhoek Hospital, The Hague, The Netherlands

7 Paediatric Immunology, Erasmus MC Sophia Children's Hospital, Rotterdam, The Netherlands

8 Paediatrics, Juliana Children's Hospital/Haga Teaching Hospital, The Hague, The Netherlands

9 Jeroen Bosch Academy, Jeroen Bosch Hospital, 'sHertogenbosch, The Netherlands

10 Tranzo, Tilburg University, Tilburg, The Netherlands

11 Immunology and Internal Medicine, Erasmus MC, Rotterdam, The Netherlands

12 Department of Radiology, Erasmus MC Sophia Children's Hospital, Rotterdam, The Netherlands

13 Radiology, Università degli Studi di Roma La Sapienza, Rome, Italy

14 Institute of Nuclear Medicine, University College London, London, UK

15 Department of Molecular Medicine, Sapienza University of Rome, Rome, Italy

16 Immunology Section Department of Medicine, Imperial College London, London, UK

17 Department of Respiratory Medicine, Royal Brompton Hospital, London, UK
18 Department of Clinical Immunology and Allergy, Faculty of Medicine, Masaryk University, St Anne's University Hospital, Brno, Czech Republic

19 Department of Radiology, Faculty of Medicine, Masaryk University, St Anne's University Hospital, Brno, Czech Republic

20 RECETOX, Faculty of Science, Masaryk University, Brno, Czech Republic

21 Radiology, Addenbrooke's Hospital, Cambridge, UK

22 Immunology, Papworth Hospital, Cambridge, UK

23 Immunology, Addenbrooke's Hospital, Cambridge, UK

24 Radiology, Papworth Hospital, Cambridge, UK

25 Paediatric Immunology, Great Ormond Street Hospital, London, UK

26 Department of Radiology, University of Brescia, Brescia, Italy

27 Pediatrics Clinic and Institute for Molecular Medicine A. Nocivelli, Department of Clinical and Experimental Sciences, University of Brescia and ASST-Spedali Civili of Brescia, Brescia, Italy

28 Pediatrics Clinic, ASST-Spedali Civili, Brescia, Italy

29 Radiology, IBB-CNR University of Naples Federico II, Naples, Italy

30 Immunology, University of Naples Federico II, Naples, Italy

31 Paediatric University Hospital, Cairo, Egypt

32 Diagnostic Radiology, Hanover Medical School, Hanover, Germany

33 Allergy, Immunology and Respiratory Medicine, The Alfred Hospital, Melbourne, Australia

34 Primary Immunodeficiency Unit, Nuffield Department of Medicine, University of Oxford, Oxford, UK

35 Immunology, Cliniques Universitaires de Bruxelles Hôpital Erasme, Brussels, Belgium

36 Paediatric Immunology and Pulmonology, University Hospitals, Leuven, Belgium 
37 Radiology, Heartlands Hospital, Birmingham, UK

38 Paediatric Immunology Department, Heartlands Hospital Birmingham, Birmingham, UK

39 UCL Respiratory Medicine, University College London, London, UK
40

ESID Registry Working Party, University Hospital Freiburg, Freiburg, Germany

41 Paediatric Radiology and Sonographics, Children's Hospital auf der Bult, Hanover, Germany

42 Radiology, Asklepios Clinic Lich, Lich, Germany 